\title{
Rheumatic manifestations of COVID-19: a systematic review and meta-analysis
}

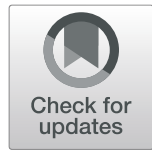

\author{
Jacopo Ciaffi ${ }^{1 *} \mathbb{D}$, Riccardo Meliconi ${ }^{1,2}$, Piero Ruscitti ${ }^{3}$, Onorina Berardicurti ${ }^{3}$, Roberto Giacomelli ${ }^{3}$ and \\ Francesco Ursini ${ }^{1,2}$
}

\begin{abstract}
Background: Different proportions of musculoskeletal or autoimmune manifestations associated with COVID-19 have been reported in literature. We performed a systematic review and meta-analysis with the aim of assessing the prevalence of rheumatic manifestations in patients affected by COVID-19, as initial symptom or during disease course.

Methods: A database search was run on May 18th, 2020, using two distinct strategies. We were interested in the percentage of symptoms of potential rheumatologic interest observed in large population studies of COVID-19 cases, and in identifying uncommon autoimmune disorders described in patients with COVID-19. For manifestations individually reported, a meta-analysis was performed taking into consideration the proportion of COVID-19 patients presenting the symptom.

Results: Eighty eight original articles were included in the systematic review and 51 in the meta-analysis. We found pooled estimates of 19\% for muscle pain and 32\% for fatigue as initial symptom of COVID-19 presentation and, respectively, of 16 and 36\% during the disease course. Only one article discussed arthralgia as unique symptom. Additionally, we found that vasculitis, chilblains, presence of autoantibodies commonly found in patients with rheumatic diseases, or autoimmune haematological and neurological disorders have all been reported in patients with COVID-19.

Conclusions: In conclusion, our review and meta-analysis emphasises that symptoms potentially leading to rheumatologic referral are common in patients with COVID-19. Therefore, COVID-19 is a new differential diagnosis to bear in mind when evaluating patients with musculoskeletal symptoms and rheumatologists might play a crucial role in identifying COVID-19 cases in early phases of the illness.
\end{abstract}

Keywords: COVID-19, Arthralgia, Myalgia, Fatigue, Rheumatology

\footnotetext{
* Correspondence: jacopo.ciaffi91@gmail.com

'Medicine \& Rheumatology Unit, IRCCS Istituto Ortopedico Rizzoli (IOR), via

Pupilli 1, 40136 Bologna, Italy

Full list of author information is available at the end of the article
}

(c) The Author(s). 2020 Open Access This article is licensed under a Creative Commons Attribution 4.0 International License, which permits use, sharing, adaptation, distribution and reproduction in any medium or format, as long as you give appropriate credit to the original author(s) and the source, provide a link to the Creative Commons licence, and indicate if changes were made. The images or other third party material in this article are included in the article's Creative Commons licence, unless indicated otherwise in a credit line to the material. If material is not included in the article's Creative Commons licence and your intended use is not permitted by statutory regulation or exceeds the permitted use, you will need to obtain permission directly from the copyright holder. To view a copy of this licence, visit http://creativecommons.org/licenses/by/4.0/. The Creative Commons Public Domain Dedication waiver (http://creativecommons.org/publicdomain/zero/1.0/) applies to the data made available in this article, unless otherwise stated in a credit line to the data. 


\section{Background}

At the end of 2019 several cases of atypical pneumonia emerged in the Chinese province of Hubei and, in January 2020, severe acute respiratory virus 2 (SARS-CoV-2) was identified as the causative agent of the novel coronavirus disease 2019 (COVID-19) [1], representing the third major coronavirus infection of the twenty-first century after severe acute respiratory syndrome (SARS) [2] and Middle East respiratory syndrome (MERS) [3]. As of August 19, 2020, 22.173.973 confirmed cases and 781.756 deaths were reported globally (https://coronavirus.jhu.edu/map.html). Spreading from country to country, COVID-19 rapidly became a key priority for the whole scientific community. Growing and compelling evidence suggests that the manifestations of COVID-19 are protean, ranging from laboratory-confirmed asymptomatic infection to critical illness with rapidly progressive respiratory distress syndrome [4]. In symptomatic cases, current literature outlines how respiratory and constitutional symptoms are frequently reported [4]. The finding of signs and symptoms of COVID-19 extending beyond the respiratory tract can be explained, at least in part, by the ubiquitous expression and tissue distribution of angiotensin-converting enzyme 2 (ACE2), the major SARS-CoV-2 entry receptor [5]. In particular, ACE-2 is found also in bowel, endothelium of small vessels, smooth muscle, skeletal muscle and even synovial tissue [6]. It is therefore not unexpected that, besides cough and dyspnoea, COVID-19 patients often experience fever, fatigue, muscle pain, or arthralgia. However, some of the symptoms caused by COVID-19 are commonly described in other diseases and are frequently reported, for instance, also in patients with rheumatic conditions. Interestingly, the association between viral infections and rheumatic diseases is already well-recognised. Viruses can be direct etiologic agents of acute and chronic arthritis [7], and of different forms of vasculitis, both in children and adults [8]. Moreover, a role in the pathogenesis of systemic sclerosis [9] and of polymyalgia rheumatica or giant-cell arteritis [10] has been proposed. Although the causal relationship between a viral trigger and rheumatic diseases is well-known to rheumatologists, when patients with musculoskeletal complaints are evaluated, the identification of an infectious aetiology can be extremely complicated as findings are often equivocal. Since symptoms of potential rheumatologic interest have been frequently reported in COVID-19 patients, the new virus outbreak represents a previously unseen differential diagnosis to be henceforth taken into consideration. However, inconsistent percentages of musculoskeletal symptoms are reported in literature. The aim of the present systematic review and meta-analysis is to provide an updated estimate of the prevalence of clinical manifestations of potential rheumatologic relevance in COVID-19, emphasising how rheumatologists might play a crucial role in identifying cases of COVID-19 presenting with extra-respiratory symptoms.

\section{Methods \\ Search strategy and study selection}

The systematic review was performed on MedLine through PubMed search. Two search strings were built. The first string was primarily aimed at identifying large cohort studies or randomised controlled trials (RCTs) reporting clinical characteristics of patients affected by COVID-19. Additionally, to ensure no relevant references describing manifestations of potential rheumatologic interest were missed, we ran a second search using specific keywords referring to rheumatic symptoms possibly related to COVID-19, as suggested by a preliminary appraisal of currently available evidence.

First search string was: ("cohort" or "observational" or "retrospective" or "prospective" or "trial" or "cross-sectional") and ("covid" or "sars-cov-2" or "novel coronavirus" or "2019-ncov") and ("symptom"* or "clinical features" or "clinical characteristic*").

Second search string was: (vasculitis" or "ulcer" or "raynaud" or "arthritis" or "acrocyanosis" or "chilblains" or "kawasaki" or "autoimmun" or "autoantibodies" or "ana" or "anti nuclear" or "antiphospholipid" or "anca" or "citrullinated" or "rheumatoid factor") and ("covid" or "sars-cov-2" or "novel coronavirus" or "2019-ncov").

No date restriction was applied and two investigators (J.C. and R.M.) worked independently to screen titles and abstracts of the literature retrieved up to 18th May 2020. Full-text evaluation was then performed, along with manual search of references to identify additional relevant papers. Disagreements were resolved through discussion with a third investigator (F.U.) when consensus could not be achieved. In drafting the final manuscript, we followed the Preferred Reporting Items for Systematic Reviews and Meta-Analyses (PRISMA) guidelines [11].

\section{Eligibility criteria}

For the first search, on the basis of preliminary scouting of literature, we hypothesized a prevalence of musculoskeletal symptoms in COVID-19 of about 20\%. Accordingly, we calculated a minimum sample size of 106 patients to estimate such proportion with $5 \%$ absolute precision and $80 \%$ confidence. On this basis, we decided to include in our systematic review and meta-analysis only studies reporting cohorts of at least 100 patients. The PICO (population, intervention, comparator, outcome) framework [12] was applied to build the search question and the inclusion/exclusion criteria. Publications written in a language other than English were excluded. All studies, published as peer-reviewed final articles and meeting the following criteria, were considered eligible: 
- Population: children or adult patients with diagnosis of COVID-19;

- Intervention: assessment of clinical characteristics at onset of COVID-19 or during its evolution;

- Comparator: the presence of a comparator was not considered necessary;

- Outcomes: percentage of patients presenting symptoms of potential rheumatologic interest.

\section{Data extraction and quality assessment}

Data were extracted and summarized by the first author (J.C.) and revised by the second author (R.M.). From each selected article, the following features were reported: first author; year of publication; origin; study design; total number of patients; hospital or non-hospital setting; presence and type of manifestation of potential rheumatologic interest, at the onset of disease or during its evolution as overall prevalence.

Quality of the included studies was assessed using the Cochrane risk of bias tool [13] for randomized trials, the Newcastle-Ottawa scale [14] for non-randomized studies, and the Johanna-Briggs Institute critical appraisal tools [15] for case reports and case series.

\section{Statistical analysis}

A meta-analysis was performed to estimate the overall proportion of COVID-19 patients presenting with a clinical manifestation of potential rheumatologic interest. Considering the substantial heterogeneity expected, we adopted a random-effects model to pool data from included studies. Forest plots were used to graphically represent the effect size, which was the pooled prevalence of the clinical manifestation of interest, as initial symptom or during COVID-19 infection, at 95\% confidence intervals. $I^{2}$ was calculated to measure between-studies heterogeneity. Publication bias was assessed with Egger's regression test after visual inspection of funnel plot [16]. In case of publication bias, the "trim and fill" method was used to re-calculate pooled estimates [17].

\section{Results}

The search strategy identified 512 articles from the first string and 307 from the second (Fig. 1a and b). Four additional papers were identified through manual search of references. Respectively from the first and the second search, 115 and 50 studies were considered potentially relevant for full text evaluation. Overall, the full article review identified 88 studies that proceeded to data extraction and analysis. Of these, 51 articles were included in the meta-analysis. Characteristics of the selected studies are shown in Table 1. Three RCTs were included and they were considered at moderate risk of bias (Table S1). Of the 59 articles evaluated through the Newcastle-Ottawa scale, 41 were rated of good quality, 10 of fair quality, and 8 of poor quality (Table S2). Finally, 15 case series and 11 case reports were deemed eligible after the application of Johanna-Briggs critical appraisal tools.

\section{Arthralgia in COVID-19}

Redd et al. [18] reported a proportion of arthralgia at presentation of $2.5 \%$ in 318 COVID-19 patients, but this was the only study describing the prevalence of arthralgia as independent symptom. Six additional articles reported the presence of arthralgia, but always combined

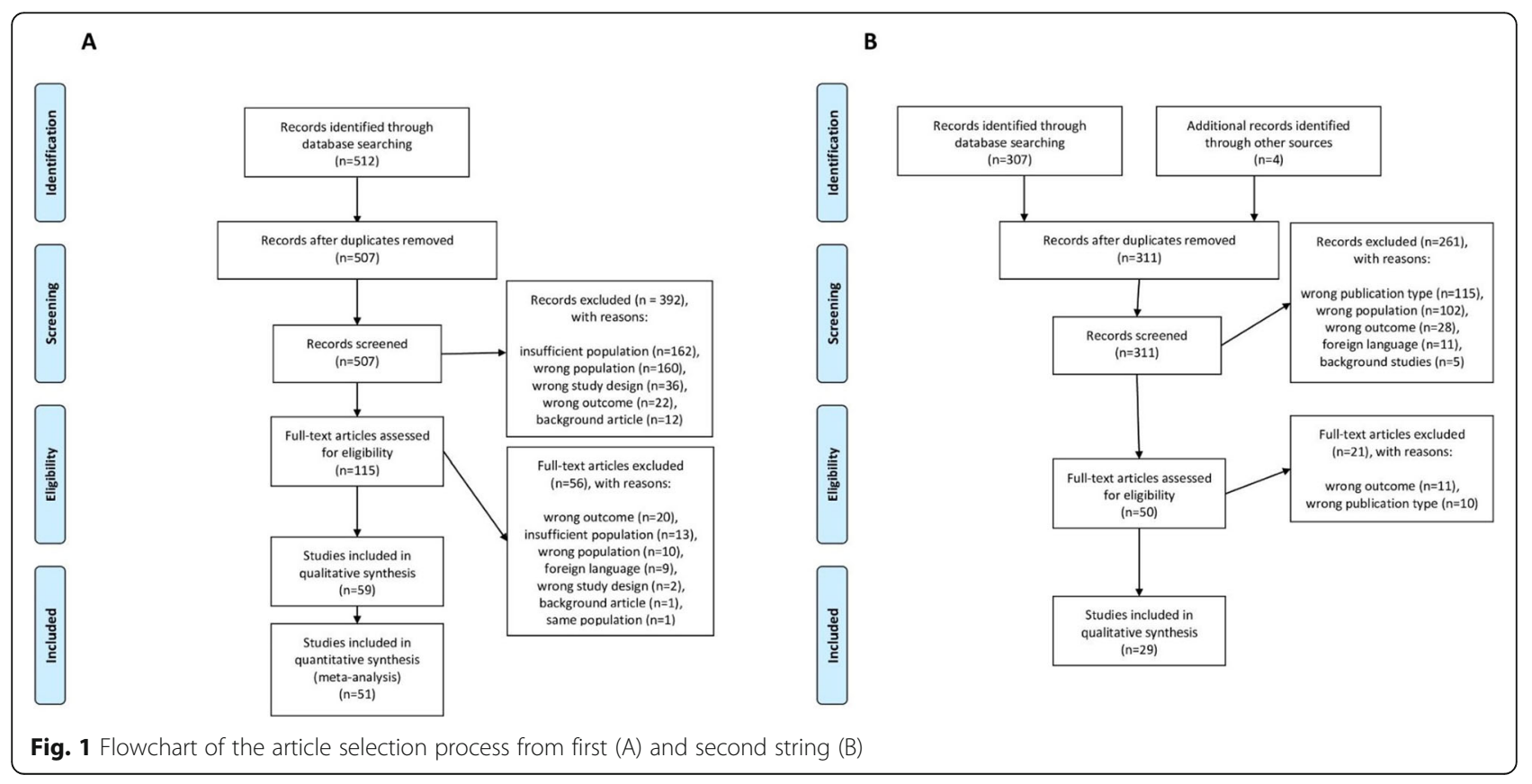


Table 1 Characteristics of the included studies

\begin{tabular}{|c|c|c|c|c|c|c|c|}
\hline Study & Year & Journal & Country & Study design & $\begin{array}{l}\text { Total } \\
\text { patients }\end{array}$ & Setting & Findings of rheumatologic interest \\
\hline Alramthan & 2020 & $\begin{array}{l}\text { Clin Exp } \\
\text { Dermatol }\end{array}$ & Kuwait & case report & 2 & non-hospital & acro-ischemic lesions \\
\hline Andina & 2020 & Pediatr Dermatol & Spain & case series & 22 & non-hospital & chilblains \\
\hline Beyrouty & 2020 & $\begin{array}{l}\text { J Neurol } \\
\text { Neurosurg } \\
\text { Psychiatry }\end{array}$ & UK & case series & 6 & hospital & antiphospholipid antibodies \\
\hline Bomhof & 2020 & Br J Haematol & Netherlands & case series & 3 & hospital & immune thrombocytopenia \\
\hline Bouaziz & 2020 & $\begin{array}{l}\text { J Eur Acad } \\
\text { Dermatol } \\
\text { Venereol }\end{array}$ & France & case series & 7 & non-hospital & chilblains \\
\hline Bowles & 2020 & NEJM & UK & case series & 35 & hospital & antiphospholipid antibodies \\
\hline Cao & 2020 & Clin Infect Dis & China & $\begin{array}{l}\text { retrospective } \\
\text { observational } \\
\text { study }\end{array}$ & 102 & hospital & myalgia (prevalence); fatigue (prevalence) \\
\hline Castelnovo & 2020 & $\begin{array}{l}\text { J Eur Acad } \\
\text { Dermatol } \\
\text { Venereol }\end{array}$ & Italy & case series & 2 & non-hospital & cutaneous vasculitis \\
\hline Chen & 2020 & $J$ Infect & China & $\begin{array}{l}\text { retrospective } \\
\text { observational } \\
\text { study }\end{array}$ & 249 & hospital & fatigue (onset) \\
\hline Chen & 2020 & Acta Ophthalmol & China & $\begin{array}{l}\text { retrospective } \\
\text { observational } \\
\text { study }\end{array}$ & 535 & non-hospital & $\begin{array}{l}\text { fatigue (prevalence); arthralgia or myalgia } \\
\text { (prevalence) }\end{array}$ \\
\hline Chen & 2020 & Infection & China & $\begin{array}{l}\text { retrospective } \\
\text { observational } \\
\text { study }\end{array}$ & 145 & hospital & myalgia (prevalence); fatigue (prevalence) \\
\hline Chen & 2020 & Chest & China & $\begin{array}{l}\text { retrospective } \\
\text { observational } \\
\text { study }\end{array}$ & 1590 & hospital & $\begin{array}{l}\text { fatigue (prevalence on } 1365 \text { patients); myalgia or } \\
\text { arthralgia (prevalence on } 1338 \text { patients) }\end{array}$ \\
\hline Chen & 2020 & $\begin{array}{l}\text { J Gerontol a Bio } \\
\text { Sci Med Sci }\end{array}$ & China & $\begin{array}{l}\text { retrospective } \\
\text { observational } \\
\text { study }\end{array}$ & 203 & hospital & $\begin{array}{l}\text { fatigue (prevalence); myalgia or arthralgia } \\
\text { (prevalence) }\end{array}$ \\
\hline Chen & 2020 & BMJ & China & $\begin{array}{l}\text { retrospective } \\
\text { observational } \\
\text { study }\end{array}$ & 274 & hospital & myalgia (onset); fatigue (onset) \\
\hline Chen & 2020 & Diabetes Care & China & $\begin{array}{l}\text { retrospective } \\
\text { observational } \\
\text { study }\end{array}$ & 904 & hospital & myalgia (onset); fatigue (onset) \\
\hline Colonna & 2020 & Pediatr Dermatol & Italy & case series & 4 & non-hospital & chilblains \\
\hline Cordoro & 2020 & Pediatr Dermatol & USA & case series & 6 & non-hospital & chilblains \\
\hline de Masson & 2020 & J Am Acad Dermat & & $\begin{array}{l}\text { retrospective } \\
\text { observational } \\
\text { study }\end{array}$ & 277 & non-hospital & chilblains \\
\hline Dogan & 2020 & $\begin{array}{l}\text { Brain Behav } \\
\text { Immun }\end{array}$ & Turkey & case series & 6 & hospital & autoimmune encephalitis \\
\hline Du & 2020 & $\begin{array}{l}\text { Ann Am Thorac } \\
\text { Soc }\end{array}$ & China & $\begin{array}{l}\text { retrospective } \\
\text { observational } \\
\text { study }\end{array}$ & 109 & hospital & myalgia (onset); fatigue (onset) \\
\hline $\begin{array}{l}\text { Galeano- } \\
\text { Valle }\end{array}$ & 2020 & Thromb Res & Spain & $\begin{array}{l}\text { retrospective } \\
\text { observational } \\
\text { study }\end{array}$ & 24 & hospital & antiphospholipid antibodies \\
\hline $\begin{array}{l}\text { Galván- } \\
\text { Casas }\end{array}$ & 2020 & $\mathrm{Br} J$ Dermatol & Spain & $\begin{array}{l}\text { retrospective } \\
\text { observational } \\
\text { study }\end{array}$ & 375 & $\begin{array}{l}\text { hospital and } \\
\text { non-hospital }\end{array}$ & $\begin{array}{l}\text { pseudo-chillblain (prevalence); livedo/necrosis: } \\
\text { (prevalence) }\end{array}$ \\
\hline Garazzino & 2020 & Euro Surveill & Italy & retrospective & 168 & hospital and & fatigue (prevalence) \\
\hline
\end{tabular}


Table 1 Characteristics of the included studies (Continued)

\begin{tabular}{|c|c|c|c|c|c|c|c|}
\hline Study & Year & Journal & Country & Study design & $\begin{array}{l}\text { Total } \\
\text { patients }\end{array}$ & Setting & Findings of rheumatologic interest \\
\hline & & & & $\begin{array}{l}\text { observational } \\
\text { study }\end{array}$ & children & non-hospital & \\
\hline Harzallah & 2020 & J Thromb Haem & France & case series & 56 & not-reported & antiphospholipid antibodies \\
\hline $\mathrm{Hu}$ & 2020 & Phytomedicine & China & $\mathrm{RCT}$ & 284 & hospital & fatigue (prevalence) \\
\hline Huang & 2020 & J Med Virol & China & $\begin{array}{l}\text { retrospective } \\
\text { observational } \\
\text { study }\end{array}$ & 344 & hospital & fatigue (prevalence) \\
\hline Huang & 2020 & $\begin{array}{l}\text { PLOS Negl Trop } \\
\text { Dis }\end{array}$ & China & $\begin{array}{l}\text { retrospective } \\
\text { observational } \\
\text { study }\end{array}$ & 202 & hospital & myalgia (onset); fatigue (onset) \\
\hline Hung & 2020 & Lancet & Hong Kong & $\mathrm{RCT}$ & 127 & hospital & myalgia (prevalence); malaise (prevalence) \\
\hline Hur & 2020 & $\begin{array}{l}\text { Otolaryngol } \\
\text { Head Neck Surg }\end{array}$ & USA & $\begin{array}{l}\text { retrospective } \\
\text { observational } \\
\text { study }\end{array}$ & 486 & hospital & fatigue (prevalence) \\
\hline Javanian & 2020 & Rom J Int Med & Iran & $\begin{array}{l}\text { retrospective } \\
\text { observational } \\
\text { study }\end{array}$ & 100 & hospital & myalgia (prevalence); fatigue (prevalence) \\
\hline $\mathrm{Ji}$ & 2020 & Epidemiol Infect & China & $\begin{array}{l}\text { retrospective } \\
\text { observational } \\
\text { study }\end{array}$ & 101 & hospital & myalgia (prevalence); fatigue (prevalence) \\
\hline Jones & 2020 & Hosp Pediatr & USA & case report & 1 & hospital & Kawasaki disease \\
\hline Klopfenstein & 2020 & Clin Res Hepato & France & $\begin{array}{l}\text { retrospective } \\
\text { observational } \\
\text { study }\end{array}$ & 114 & $\begin{array}{l}\text { hospital and } \\
\text { non-hospital }\end{array}$ & fatigue (prevalence) \\
\hline Kolivras & 2020 & JAAD Case Rep & USA & case report & 1 & non-hospital & chilblains \\
\hline Lazarian & 2020 & Br J Haematol & France & case series & 7 & hospital & autoimmune hemolytic anemia \\
\hline $\mathrm{Li}$ & 2020 & Clin Infect Dis & China & $\begin{array}{l}\text { retrospective } \\
\text { observational } \\
\text { study }\end{array}$ & 105 & hospital & fatigue (prevalence) \\
\hline $\mathrm{Li}$ & 2020 & Br J Haematol & USA & case report & 1 & hospital & Evans syndrome \\
\hline Lian & 2020 & Clin Infect Dis & China & $\begin{array}{l}\text { retrospective } \\
\text { observational } \\
\text { study }\end{array}$ & 788 & hospital & myalgia (prevalence); fatigue (prevalence) \\
\hline Liguori & 2020 & Brain Behav Im & Italy & $\begin{array}{l}\text { retrospective } \\
\text { observational } \\
\text { study }\end{array}$ & 103 & hospital & myalgia (prevalence); fatigue (prevalence) \\
\hline Liu & 2020 & J Clin Virol & China & $\begin{array}{l}\text { retrospective } \\
\text { observational } \\
\text { study }\end{array}$ & 140 & hospital & myalgia (onset); fatigue (onset) \\
\hline Liu & 2020 & $J$ Infect & China & $\begin{array}{l}\text { retrospective } \\
\text { observational } \\
\text { study }\end{array}$ & 245 & hospital & myalgia (prevalence); fatigue (prevalence) \\
\hline Lopez & 2020 & Br J Haematol & USA & case report & 1 & hospital & autoimmune hemolytic anemia \\
\hline $\mathrm{Lu}$ & 2020 & Pediatr Infect & China & $\begin{array}{l}\text { retrospective } \\
\text { observational } \\
\text { study }\end{array}$ & $\begin{array}{l}110 \\
\text { children }\end{array}$ & hospital & fatigue (prevalence) \\
\hline Meng & 2020 & Plos Pathog & China & $\begin{array}{l}\text { retrospective } \\
\text { observational } \\
\text { study }\end{array}$ & 168 & hospital & myalgia (prevalence); fatigue (prevalence) \\
\hline Menter & 2020 & Histopathology & Switzerland & case series & 21 & post-mortem & vasculitis of the pulmonary veins and capillaries \\
\hline Mo & 2020 & Clin Infect Dis & China & $\begin{array}{l}\text { retrospective } \\
\text { observational } \\
\text { study }\end{array}$ & 155 & hospital & $\begin{array}{l}\text { fatigue (prevalence); myalgia or arthralgia } \\
\text { (prevalence) }\end{array}$ \\
\hline Moeinzadeh & 2020 & Iran J Kidney Dis & Iran & case report & 1 & hospital & glomerulonephritis and ANCA positivity \\
\hline
\end{tabular}


Table 1 Characteristics of the included studies (Continued)

\begin{tabular}{|c|c|c|c|c|c|c|c|}
\hline Study & Year & Journal & Country & Study design & $\begin{array}{l}\text { Total } \\
\text { patients }\end{array}$ & Setting & Findings of rheumatologic interest \\
\hline Nowak & 2020 & Pol Arch Intern & Poland & $\begin{array}{l}\text { retrospective } \\
\text { observational } \\
\text { study }\end{array}$ & 169 & hospital & fatigue (prevalence) \\
\hline Paderno & 2020 & $\begin{array}{l}\text { Int Forum } \\
\text { Allergy Rhinol }\end{array}$ & Italy & $\begin{array}{l}\text { retrospective } \\
\text { observational } \\
\text { study }\end{array}$ & 295 & $\begin{array}{l}\text { hospital and } \\
\text { non-hospital }\end{array}$ & arthromyalgia (prevalence and onset) \\
\hline Palaiodimos & 2020 & Metabolism & USA & $\begin{array}{l}\text { retrospective } \\
\text { observational } \\
\text { study }\end{array}$ & 200 & hospital & myalgia (onset) \\
\hline Pan & 2020 & $\begin{array}{l}\text { Am J } \\
\text { Gastroenterol }\end{array}$ & China & $\begin{array}{l}\text { retrospective } \\
\text { observational } \\
\text { study }\end{array}$ & 103 & hospital & myalgia (prevalence) \\
\hline Pilotto & 2020 & Ann Neurol & Italy & case report & 1 & hospital & autoimmune encephalitis \\
\hline Qi & 2020 & Int J Infect Dis & China & $\begin{array}{l}\text { retrospective } \\
\text { observational } \\
\text { study }\end{array}$ & 147 & hospital & fatigue (prevalence) \\
\hline Redd & 2020 & Gastroenterology & USA & $\begin{array}{l}\text { retrospective } \\
\text { observational } \\
\text { study }\end{array}$ & 318 & hospital & myalgia (onset); fatigue (onset); arthralgia (onset) \\
\hline Ren & 2020 & Intensive Care & China & $\begin{array}{l}\text { retrospective } \\
\text { observational } \\
\text { study }\end{array}$ & 150 & hospital & myalgia (prevalence); fatigue (prevalence) \\
\hline $\begin{array}{l}\text { Rivera- } \\
\text { Figueroa }\end{array}$ & 2020 & Indian Pediatr & India & case report & 1 & hospital & Kawasaki disease \\
\hline Sedaghat & 2020 & J Clin Neurosci & Iran & case report & 1 & hospital & Guillain Barre syndrome \\
\hline Shi & 2020 & Diabetes Care & China & $\begin{array}{l}\text { retrospective } \\
\text { observational } \\
\text { study }\end{array}$ & 306 & hospital & myalgia (prevalence); fatigue (prevalence) \\
\hline Shi & 2020 & JAMA Cardiol & China & $\begin{array}{l}\text { retrospective } \\
\text { observational } \\
\text { study }\end{array}$ & 416 & hospital & myalgia (prevalence); fatigue (prevalence) \\
\hline Suarez-Valle & 2020 & $\begin{array}{l}\text { J Eur Acad } \\
\text { Dermatol } \\
\text { Venereol }\end{array}$ & Spain & case series & 3 & hospital & acro-ischemic lesions \\
\hline Tang & 2020 & BMJ & China & $\mathrm{RCT}$ & 150 & hospital & fatigue (prevalence on 136 pts) \\
\hline Tian & 2020 & $J$ infect & China & $\begin{array}{l}\text { retrospective } \\
\text { observational } \\
\text { study }\end{array}$ & 262 & hospital & fatigue (prevalence) \\
\hline $\begin{array}{l}\text { Vanegas- } \\
\text { Ramirez }\end{array}$ & 2020 & $\begin{array}{l}\text { J Eur Acad } \\
\text { Dermatol } \\
\text { Venereol }\end{array}$ & Germany & case report & 1 & hospital & vasculitis \\
\hline Verdoni & 2020 & Lancet & Italy & $\begin{array}{l}\text { retrospective } \\
\text { observational } \\
\text { study }\end{array}$ & 10 & hospital & Kawasaki disease \\
\hline Wang & 2020 & JAMA & China & $\begin{array}{l}\text { retrospective } \\
\text { observational } \\
\text { study }\end{array}$ & 138 & hospital & myalgia (prevalence); fatigue (prevalence) \\
\hline Wang & 2020 & Crit Care & China & $\begin{array}{l}\text { retrospective } \\
\text { observational } \\
\text { study }\end{array}$ & 107 & hospital & myalgia (prevalence); fatigue (prevalence) \\
\hline Wang & 2020 & J Med Virol & China & $\begin{array}{l}\text { retrospective } \\
\text { observational } \\
\text { study }\end{array}$ & 889 & non-hospital & myalgia (prevalence); fatigue (prevalence) \\
\hline Wang & 2020 & Int $J \operatorname{lnf}$ Dis & China & $\begin{array}{l}\text { retrospective } \\
\text { observational } \\
\text { study }\end{array}$ & 125 & hospital & myalgia (prevalence); fatigue (prevalence) \\
\hline
\end{tabular}


Table 1 Characteristics of the included studies (Continued)

\begin{tabular}{|c|c|c|c|c|c|c|c|}
\hline Study & Year & Journal & Country & Study design & $\begin{array}{l}\text { Total } \\
\text { patients }\end{array}$ & Setting & Findings of rheumatologic interest \\
\hline Wang & 2020 & $\begin{array}{l}\text { Diabetes Res } \\
\text { Clin }\end{array}$ & China & $\begin{array}{l}\text { retrospective } \\
\text { observational } \\
\text { study }\end{array}$ & 132 & hospital & myalgia (prevalence); fatigue (prevalence) \\
\hline Wu & 2020 & $\begin{array}{l}\text { JAMA Intern } \\
\text { Med }\end{array}$ & China & $\begin{array}{l}\text { retrospective } \\
\text { observational } \\
\text { study }\end{array}$ & 201 & hospital & myalgia or fatigue (onset) \\
\hline Yan & 2020 & $\begin{array}{l}\text { BMJ open } \\
\text { diabetes res care }\end{array}$ & China & $\begin{array}{l}\text { retrospective } \\
\text { observational } \\
\text { study }\end{array}$ & 193 & hospital & fatigue (prevalence) \\
\hline Yang & 2020 & $J$ Infect & China & $\begin{array}{l}\text { retrospective } \\
\text { observational } \\
\text { study }\end{array}$ & 149 & hospital & myalgia (prevalence) \\
\hline Yao & 2020 & Pol Arch Intern & China & $\begin{array}{l}\text { retrospective } \\
\text { observational } \\
\text { study }\end{array}$ & 108 & hospital & myalgia or fatigue (prevalence) \\
\hline Zhang & 2020 & $J$ Infect Dis & China & $\begin{array}{l}\text { retrospective } \\
\text { observational } \\
\text { study }\end{array}$ & 112 & hospital & myalgia (prevalence); fatigue (prevalence) \\
\hline Zhang & 2020 & $\begin{array}{l}\text { Diabetes Obes } \\
\text { Metab }\end{array}$ & China & $\begin{array}{l}\text { retrospective } \\
\text { observational } \\
\text { study }\end{array}$ & 166 & hospital & myalgia (prevalence); fatigue (prevalence) \\
\hline Zhang & 2020 & NEJM & China & case series & 3 & hospital & antiphospholipid antibodies \\
\hline Zhang & 2020 & J Clin Virol & China & $\begin{array}{l}\text { retrospective } \\
\text { observational } \\
\text { study }\end{array}$ & 221 & hospital & fatigue (prevalence) \\
\hline Zhang & 2020 & J Clin Virol & China & $\begin{array}{l}\text { retrospective } \\
\text { observational } \\
\text { study }\end{array}$ & 111 & hospital & myalgia (prevalence); fatigue (prevalence) \\
\hline Zhang & 2020 & Eur Radiol & China & $\begin{array}{l}\text { retrospective } \\
\text { observational } \\
\text { study }\end{array}$ & 120 & hospital & myalgia or fatigue (onset) \\
\hline Zhao & 2020 & $\begin{array}{l}\text { AJR Am J } \\
\text { Roentgerol }\end{array}$ & China & $\begin{array}{l}\text { retrospective } \\
\text { observational } \\
\text { study }\end{array}$ & 101 & hospital & myalgia or fatigue (onset) \\
\hline Zheng & 2020 & $\begin{array}{l}\text { Eur Rev. Med } \\
\text { Pharmacol Sci }\end{array}$ & China & $\begin{array}{l}\text { retrospective } \\
\text { observational } \\
\text { study }\end{array}$ & 161 & hospital & myalgia (prevalence); fatigue (prevalence) \\
\hline Zheng & 2020 & $\begin{array}{l}\text { Clin Chem Lab } \\
\text { Med }\end{array}$ & China & $\begin{array}{l}\text { retrospective } \\
\text { observational } \\
\text { study }\end{array}$ & 141 & hospital & fatigue (prevalence) \\
\hline Zhou & 2020 & Lancet & China & $\begin{array}{l}\text { retrospective } \\
\text { observational } \\
\text { study }\end{array}$ & 191 & hospital & myalgia (prevalence); fatigue (prevalence) \\
\hline Zhou & 2020 & Eur Radiol & China & $\begin{array}{l}\text { retrospective } \\
\text { observational } \\
\text { study }\end{array}$ & 100 & hospital & myalgia (prevalence); fatigue (prevalence) \\
\hline Zhou & 2020 & Clin Exp Hypert & China & $\begin{array}{l}\text { retrospective } \\
\text { observational } \\
\text { study }\end{array}$ & 110 & hospital & myalgia (prevalence); fatigue (prevalence) \\
\hline Zhou & 2020 & Plos One & China & $\begin{array}{l}\text { retrospective } \\
\text { observational } \\
\text { study }\end{array}$ & 366 & hospital & $\begin{array}{l}\text { fatigue (prevalence); myalgia and arthralgia } \\
\text { (prevalence) }\end{array}$ \\
\hline Zhou & 2020 & Clin Trans Sci & China & case series & 6 & hospital & ANA and ENA \\
\hline Zulfiqar & 2020 & NEJM & France & case report & 1 & hospital & immune thrombocytopenic purpura \\
\hline
\end{tabular}


with myalgia. Therefore, we deemed the symptom "arthralgia" not suitable for meta-analysis.

Paderno et al. [19] showed that 9.4\% of COVID-19 patients complained of arthromyalgia at disease onset, while the overall prevalence was $50.4 \%$. Zhou et al. [20] reported that only $3.8 \%$ of 366 hospitalized patients had myalgia or arthralgia, while Chen et al. [21] described a proportion of $17.5 \%$ in 1338 patients. In a cohort of 203 hospitalized patients, Chen and colleagues [22] observed that myalgia or arthralgia were present in $26.6 \%$ of cases and, describing the characteristics of 535 hospitalized and non-hospitalized COVID-19 patients, Chen et al. [23] found arthralgia or myalgia in $29 \%$ of cases, whereas Mo et al. [24] reported a prevalence of $61 \%$.

\section{Myalgia in COVID-19}

Thirty-three articles describing the proportion of COVID-19 patients experiencing myalgia were included in the meta-analysis. Of these, 7 explored myalgia at disease onset $[18,25-30]$ and 26 during COVID-19 evolution [31-56].

Pooled estimate of muscle pain as initial symptom was 0.187 (95\% CI $0.119-0.282, p<0.001$ ) (Fig. 2). The $I^{2}$ was calculated to be $95.2 \%$. Visual inspection of funnel plot (Fig. S1) suggested potential publication bias. Hence, we applied the "trim and fill" method to adjust funnel plot asymmetry and correct the variance. Two studies were removed and, after the imputed fills were added, the adjusted point estimate remained unchanged.

Pooled estimate of the prevalence of muscle pain in patients with COVID-19 was 0.156 (95\% CI 0.116-0.206) (Fig. 3). The $I^{2}$ was calculated to be $94.3 \%$. Funnel plot is shown in Fig. S2. The "trim and fill" procedure excluded two studies and, filling the missing effect sizes, resulted in an adjusted value of 0.172 (95 CI 0.129-0.226).

Moreover, 4 additional papers reported the proportion of patients experiencing myalgia combined with fatigue. At disease onset the prevalence of both symptoms aggregated varied between 16.9\% [57], 32.3\% [58], and $48 \%$ [59]. Furthermore, Yao et al. [60] described a prevalence of $25.9 \%$ during the course of the illness.

\section{Fatigue in COVID-19}

Forty-seven articles describing the percentage of COVID-19 patients complaining of fatigue were included in the meta-analysis. Of these, 7 explored fatigue at disease onset $[18,25-29,61]$ and 40 during its evolution [20-24, 31, 32, 34-39, 41-48, 50-56, 62-73].

Pooled estimate of fatigue as initial symptom was 0.317 (95\% CI 0.198-0.464) (Fig. 4). An $I^{2}$ of $97.4 \%$ was calculated. Visual inspection of funnel plot (Fig. S3) suggested no potential publication bias. Pooled estimate of prevalence of fatigue in patients with COVID-19 was 0.356 (95\% CI 0.297-0.420) (Fig. 5). The $I^{2}$ was $97.1 \%$. No plot (Fig. S4) asymmetry was detected.

Additionally, Garazzino et al. [74] and Lu et al. [75] reported prevalence of fatigue respectively of 1.8 and 3.6\% in children affected by COVID-19. These latter studies were not included in the meta-analysis as the only ones not describing data of an adult population.

\section{Vasculitis in COVID-19}

We found 7 articles reporting the occurrence of vasculitis in COVID-19 patients. With the exception of the study by Menter et al. [76], all articles were case series or case reports describing only patients with vasculitis. Verdoni et al. [77] described an increased incidence of Kawasaki disease, a medium-sized-vessel vasculitis, after the appearance of SARS-CoV-2 in the Italian province of Bergamo. The authors reported data about 10 recently diagnosed patients (age range 2.9 to 16 years, mean age $7.5 \pm 3.5$ years) with Kawasaki vasculitis, $80 \%$ of them also having positive serology for SARS-CoV-2, and presenting with clinical and biochemical characteristics different from those observed in the historical Kawasaki cohort of that region. Other two cases of Kawasaki

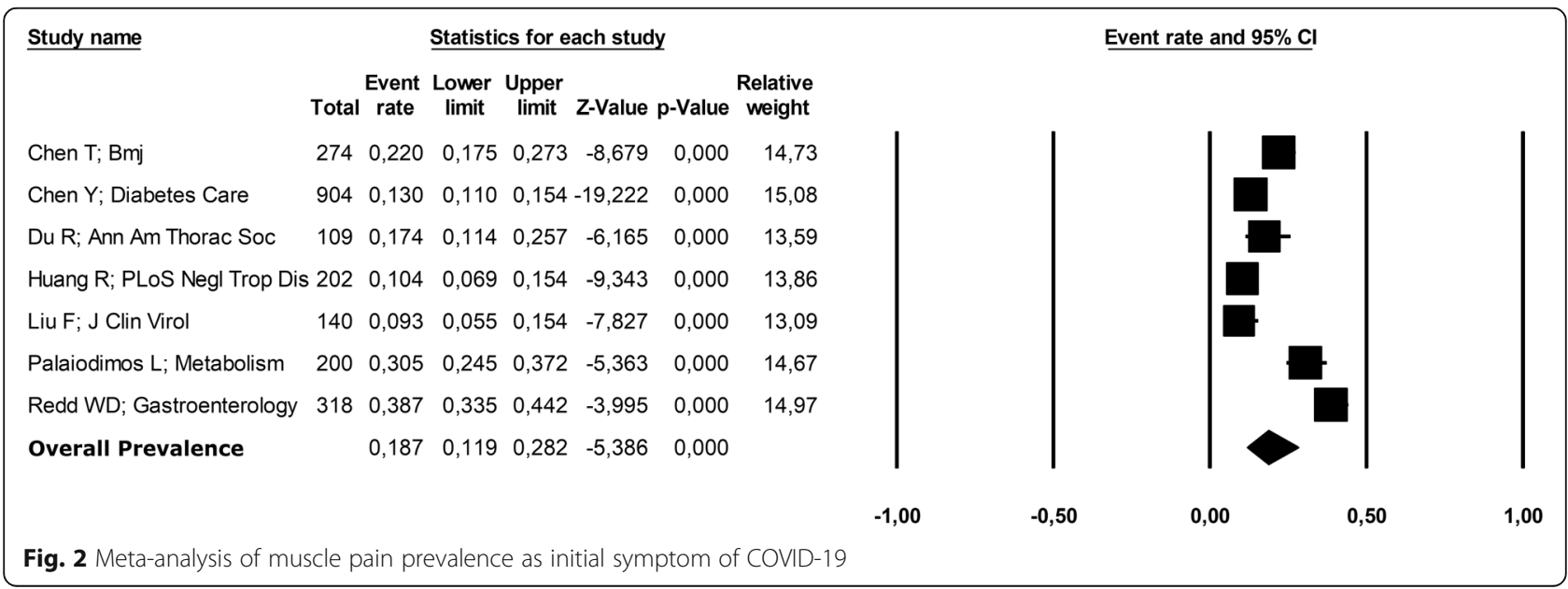




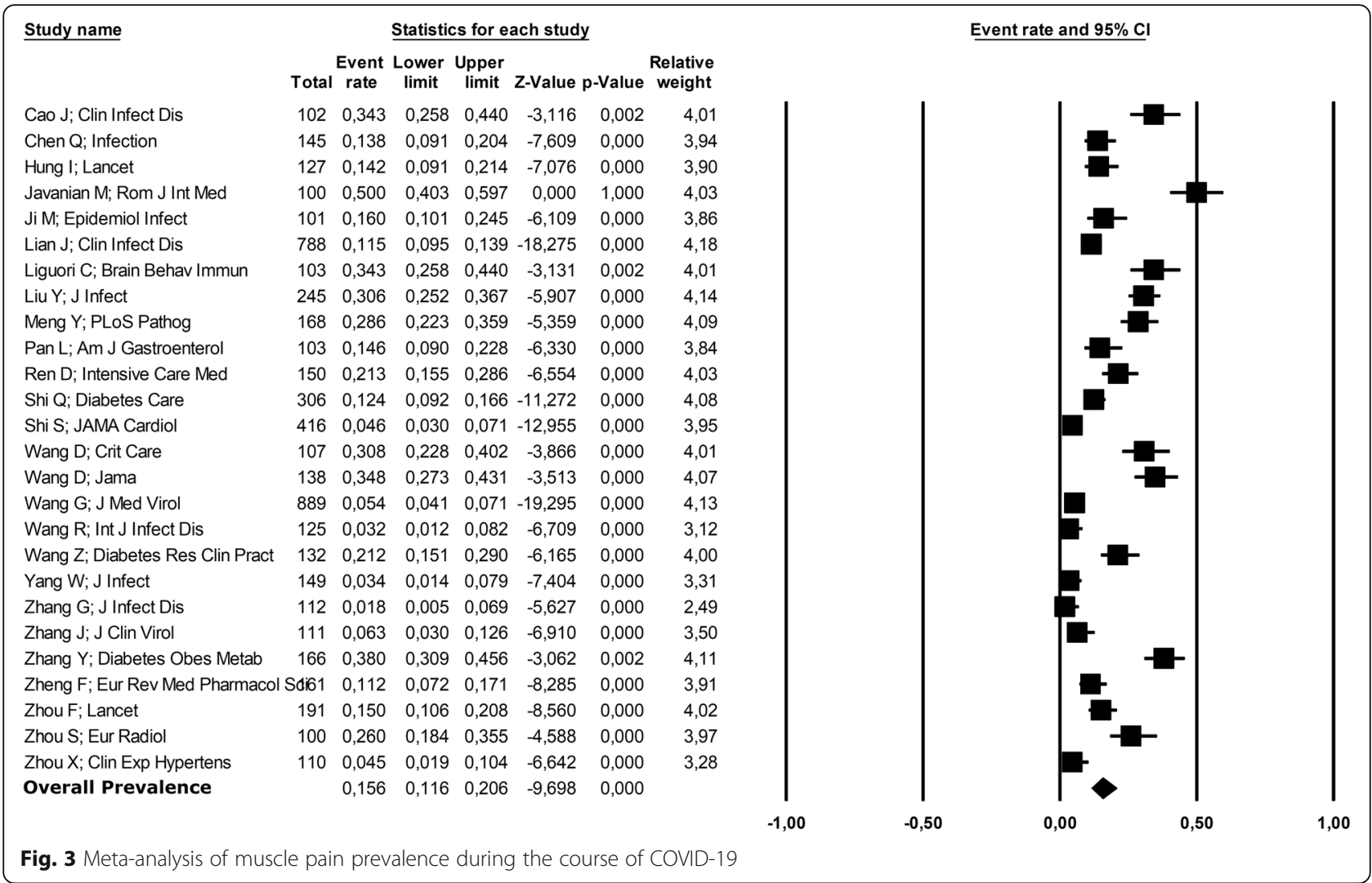

disease in paediatric age related to COVID-19 were shown by Rivera-Figueroa et al. [78] and by Jones et al. [79], while Menter et al. [76] described post-mortem findings of 21 adult COVID-19 patients revealing, in one case, florid vasculitis of pulmonary veins and capillaries. Moreover, Vanegas-Ramirez et al. [80] observed a skin rash and vasculitis in a 57-year-old COVID-19 patient, Castelnovo et al. [81] discussed 2 cases of cutaneous vasculitis in young COVID-19 patients, and Moeinzadeh et al. [82] reported the case of a 25-year-old COVID-19 patient presenting with glomerulonephritis and positive test for anti-neutrophilic cytoplasmic antibodies (ANCA).

\section{Chilblains in COVID-19}

We included 9 articles reporting the presence of chilblains in COVID-19. Galván-Casas et al. [83] presented data about the classification of lesions in acral areas of 375 COVID-19 patients, outlining a prevalence of $19 \%$ for the pseudo-chilblain pattern. Investigating $277 \mathrm{pa}-$ tients during the COVID-19 outbreak, De Masson et al. [84] described the presence of 106 chilblain lesions, although only $9 \%$ of patients had a positive test for SARSCoV-2. Similarly, Kolivras et al. [85] observed, in a case report, a 23-year-old man of chilblains induced by COVID-19 and Cordoro et al. [86] reported a case series of 6 paediatric age patients with chilblains as cutaneous

\begin{tabular}{|c|c|c|c|c|c|c|c|}
\hline \multirow[t]{2}{*}{ Study name } & \multirow[b]{2}{*}{ Total } & \multicolumn{5}{|c|}{ Statistics for each study } & \multirow[b]{2}{*}{$\begin{array}{r}\text { Relative } \\
\text { weight }\end{array}$} \\
\hline & & $\begin{array}{c}\text { Event } \\
\text { rate }\end{array}$ & $\begin{array}{l}\text { Lower } \\
\text { limit }\end{array}$ & $\begin{array}{l}\text { Upper } \\
\text { limit }\end{array}$ & Z-Value & p-Value & \\
\hline Chen J; J Infect & 249 & 0,157 & 0,117 & 0,208 & $-9,648$ & 0,000 & 14,20 \\
\hline Chen T; BMJ & 274 & 0,500 & 0,441 & 0,559 & 0,000 & 1,000 & 14,52 \\
\hline Chen Y; Diabetes Care & 904 & 0,235 & 0,208 & 0,264 & $-15,047$ & 0,000 & 14,70 \\
\hline Du R; Ann Am Thorac Soc & 109 & 0,532 & 0,438 & 0,623 & 0,668 & 0,504 & 14,08 \\
\hline Huang R; PLoS NegI Trop Dis & 202 & 0,218 & 0,166 & 0,280 & $-7,496$ & 0,000 & 14,23 \\
\hline Liu F; J Clin Virol & 140 & 0,150 & 0,100 & 0,219 & $-7,329$ & 0,000 & 13,71 \\
\hline Redd WD; Gastroenterology & 318 & 0,575 & 0,520 & 0,628 & 2,665 & 0,008 & 14,56 \\
\hline Overall Prevalence & & 0,317 & 0,198 & 0,464 & $-2,408$ & 0,016 & \\
\hline
\end{tabular}

Fig. 4 Meta-analysis of fatigue prevalence as initial symptom of COVID-19
Event rate and $95 \% \mathrm{Cl}$

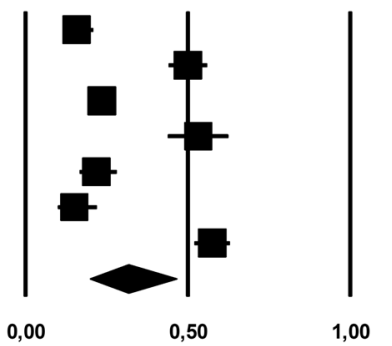




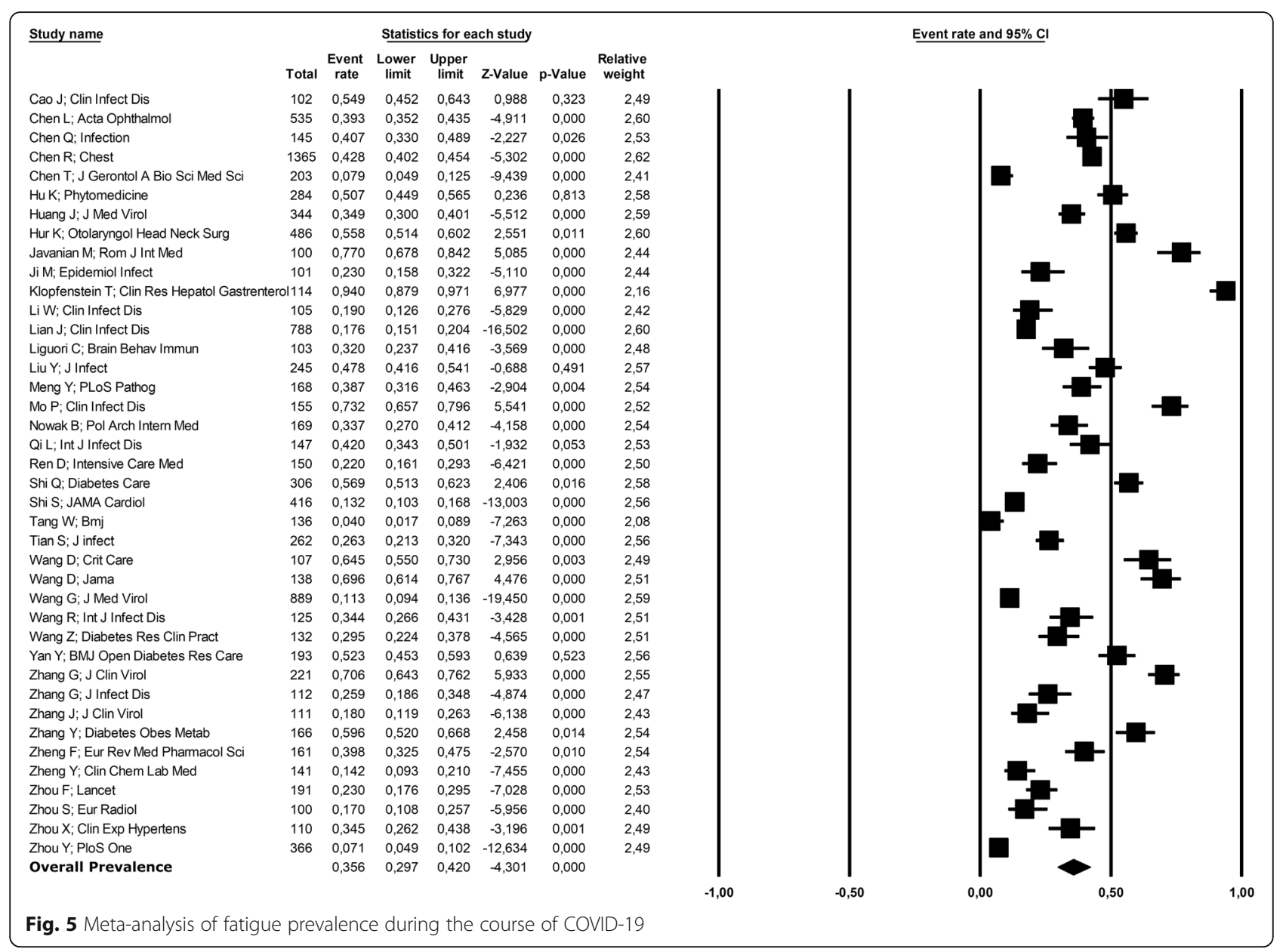

reaction to SARS-CoV-2. Colonna et al. [87] described in a case series the finding of chilblains in 4 children suspected for COVID-19, while Bouaziz et al. [88] reported chilblains in 2 of the 14 studied COVID-19 patients and Andina et al. [89] described the case series of 22 children presenting with chilblains during the COVID outbreak, although only one tested positive. Finally, Suarez-Valle et al. [90] and Alramthan et al. [91] described respectively 3 and 2 COVID-19 patients with acro-ischemic lesions.

\section{Autoantibodies in COVID-19}

We found 6 articles describing the presence of autoantibodies in patients with COVID-19. Zhou et al. [92] found a prevalence of $20 \%$ for anti-52 $\mathrm{kDa}$ SSA/Ro antibody, of $25 \%$ for anti- $60 \mathrm{kDa}$ SSA/Ro antibody and of $50 \%$ for antinuclear antibody in their cases. Zhang et al. [93] described a case series of three patients with positive antiphospholipid antibodies (aPL), and in particular anticardiolipin IgA, anti- $\beta 2$-glycoprotein I IgA and IgG. Out of 24 cases, Galeano-Valle et al. [94], studying 24 patients hospitalized with COVID-19 and venous thromboembolism, found 2 patients weakly positive for anticardiolipin IgM and anti- $\beta 2$-glycoprotein I IgM, while anticardiolipin IgG and anti- $\beta 2$-glycoprotein I IgG were negative in all patients. Harzallah et al. [95] studied 56 COVID-19 cases showing that $45 \%$ were lupus anticoagulant (LAC) positive whereas in $10 \%$ anticardiolipin or anti- 32 -glycoprotein I IgG and IgM were detected. Similarly, Bowles et al. [96] found a positive LAC in 91\% of the 35 studied COVID-19 patients with prolonged activated partial-thromboplastin time (aPTT). Moreover, in a small case series, Beyrouti et al. [97] reported that 5 of 6 patients had a positive LAC, one with medium-titre $\operatorname{IgM}$ anticardiolipin and low-titre IgG and IgM anti- $\beta 2$ glycoprotein 1 antibodies.

\section{Haematological manifestations of COVID-19 of potential rheumatologic interest}

We retrieved 5 articles assessing haematological manifestation of COVID-19 of potential rheumatologic interest. All articles were case series or case reports describing only patients with the specific haematological conditions. Lazarian et al. [98] and Lopez et al. [99] showed the occurrence of autoimmune haemolytic anaemia respectively in 7 and 1 patients affected by COVID-19. Bomhof et al. 
[100] found three cases of immune-mediated thrombocytopenia related to COVID-19 while Zulfiqar et at [101]. observed the occurrence of immune thrombocytopenic purpura in a patient with COVID-19 and Li et al. [102] reported a patient with Evans syndrome, which is characterized by a combination of autoimmune haemolytic anaemia and immune thrombocytopenia.

\section{Neurological manifestations of COVID-19 of potential rheumatologic interest}

Three articles describing neurological manifestations of COVID-19 of potential rheumatologic interest were included. All articles were case series or case reports describing only patients with the specific neurological conditions. Six cases of autoimmune encephalitis were reported by Dogan et al. [103] and an additional case was presented by Pilotto et al. [104]. Moreover, Sedaghat et al. [105] described the case of a COVID-19 patient developing Guillain-Barre syndrome.

\section{Discussion}

We performed a systematic review and meta-analysis with the aim of assessing the occurrence of rheumatic manifestations in patients affected by COVID-19. The recent SARS-CoV-2 pandemic resulted in an exceptional literature contribution; therefore, we were able to include in our review 88 original references, all published as final, peer-reviewed articles, in the last few weeks. Unfortunately, we retrieved only one article describing the prevalence of arthralgia as discrete symptom at disease onset, while 6 additional studies showed the prevalence of arthralgia combined with myalgia, with percentages ranging from $3.8 \%$ [20] to $61 \%$ [24].

Our meta-analysis shows that muscle pain and fatigue are present respectively in 19 and $32 \%$ of patients as initial presentation of COVID-19, while the overall prevalence estimates are 16 and 36\% throughout the course of the illness. Moreover, we found additional studies focusing on less common musculoskeletal or autoimmune manifestations of COVID-19, of potential interest for the rheumatologist. Vasculitis, chilblains, presence of autoantibodies commonly found in patients with rheumatic diseases, or autoimmune haematological and neurological disorders have all been reported in patients with COVID-19, although evidence from large cohort studies is still lacking.

Focusing the attention on the only two items we were able to meta-analyse in our review, it is crucial to point out how muscle pain and fatigue are among the most frequent complains in patients with rheumatic diseases. For instance, muscle pain is reported in $16 \%$ of patients with rheumatoid arthritis [106] and in up to $100 \%$ of polymyalgia rheumatica cases [107], not necessarily accompanied by stiffness [106]. Similarly, 30-35\% of patients with dermatomyositis/polymyositis [108, 109] have myalgia, but the proportion rises to $74 \%$ in newly diagnosed cases [109]. Comparable figures were observed by Noda et al. [110], with $71 \%$ of patients with dermatomyositis and $25 \%$ with polymyalgia rheumatica complaining of muscle pain. However, myalgia is a highly-reported symptom even in other connective tissue diseases. In systemic sclerosis, the frequency of muscle pain varies from 20 to $86 \%[111,112]$ and it is between 40 and $80 \%$ in systemic lupus erythematosus [113]. In vasculitis, $48 \%$ of patients with microscopic polyangitis have been reported to complain of muscle pain [114], while different cases of myalgia as initial symptom of polyarteritis nodosa [115] or of ANCA-associated vasculitis [116] have been described. Moreover, malaise or fatigue are reported in about $30 \%$ of patients with polymyalgia rheumatica [107] and $42-69 \%$ of people with rheumatoid arthritis [117]. In an international survey including over 6000 participants [118], patients with rheumatoid arthritis, systemic lupus erythematosus, ankylosing spondylitis, Sjögren's syndrome, psoriatic arthritis, or systemic sclerosis, presented severe fatigue in 41 to $57 \%$ of cases. Even higher figures were observed in systemic sclerosis by Richards et al., with $75 \%$ of patients complaining of fatigue [119], or in systemic lupus erythematosus, where fatigue was identified as one of the primary symptoms in $53-80 \%$ of patients [120]. Similarly, in ANCA-associated vasculitis, fatigue is considered a common symptom, reported in $75 \%$ of patients [121]. Finally, Grayson et al. assessed fatigue through a dedicated scale, the "multidimensional fatigue inventory 20 ", in 692 patients with 9 different forms of systemic vasculitis, observing that $76 \%$ of cases had a score $>13$, indicative of severe fatigue [122].

Arthralgia, myalgia and fatigue are the most common symptoms leading to referral of patients to a rheumatologist. As outlined by our systematic review and metaanalysis, 19\% of COVID-19 cases might present muscle pain as initial symptom, while $32 \%$ might present fatigue. It is therefore conceivable that, especially for individuals with non-specific or mild complaints and without respiratory distress, a proportion of COVID-19 patients might be referred to the rheumatologist early in the disease course. Rheumatologists should hereafter bear in mind COVID-19 as a possible differential diagnosis.

However, some limitations must be considered. Although we were able to include a considerable number of studies in our review, knowledge about COVID-19 is a rapidly evolving process and the global pandemic represents a constantly expanding field of research, with new data contributed daily. As such, our work provides preliminary information, that will need to be implemented and confirmed by forthcoming research. In this view, future studies with longitudinal follow-up of 
COVID-19 patients would provide useful data for a research agenda which, in our opinion, should address the following issues: (a) geographical differences in prevalence and characteristics of COVID-19 manifestations of potential rheumatologic interest; (b) clinical persistence and evolution of symptoms as arthralgia, myalgia and fatigue after resolution of the acute infection; (c) need for long-term follow-up and, where appropriate, treatment of COVID-19 manifestations of potential rheumatologic interest; (d) monitoring patients for possible late-onset post-infective complications of potential rheumatologic interest.

A second limitation of our study is for instance the geographic origin of the included literature. The majority of retrieved articles were contributed from a single country, China, consistently with the first identification site of the novel coronavirus and the consequent interest of the Chinese scientific community towards the outbreak, but representing a source of bias and preventing the possibility to confidently generalize our findings to other populations, particularly of non-Asian ancestry. Besides that, evidence from non-hospitalized patients was limited and the clinical characteristics of cases with different disease severity were not homogeneously reported. As a result, we could not perform metaregression or subgroup analysis to evaluate the effect of different setting of COVID-19 care or the peculiarities between critically-ill and mildly diseased patients. Finally, arthralgia as a unique symptom was poorly represented and we could not meta-analyse it.

\section{Conclusions}

In conclusion, our systematic review and meta-analysis suggests that symptoms of potential rheumatologic interest are frequently reported in COVID-19, both at onset or throughout the disease course. Accordingly, as implication for clinical practice, we would raise the awareness on the possibility that the new global threat might show up in the rheumatology office.

\footnotetext{
Abbreviations

SARS-CoV-2: Severe acute respiratory virus 2; COVID-19: Coronavirus disease 2019; SARS: Severe acute respiratory syndrome SARS; MERS: Middle East respiratory syndrome; ACE2: Angiotensin-converting enzyme 2; RCTs: Randomised controlled trials; PRISMA: Preferred Reporting Items for Systematic Reviews and Meta-Analyses; ANCA: Anti-neutrophilic cytoplasmic antibodies; aPL: Antiphospholipid antibodies; LAC: Lupus anticoagulant; aPTT: Activated partial-thromboplastin time

\section{Acknowledgements}

Not applicable.

\section{Authors' contributions}

J.C.: conception and design of the work, interpretation of data, analysis of the results, drafting and revision of the manuscript. R.M.: conception and design of the work, interpretation of data, analysis of the results, drafting and revision of the manuscript. P.R.: conception and design of the work, interpretation of data, analysis of the results, drafting and revision of the manuscript. O.B.: conception and design of the work, interpretation of data,
}

analysis of the results, drafting and revision of the manuscript. R.G.: conception and design of the work, interpretation of data, analysis of the results, drafting and revision of the manuscript. F.U.: conception and design of the work, interpretation of data, analysis of the results, drafting and revision of the manuscript. The authors have read and approved the manuscript.

\section{Funding}

The authors received no specific funding for this work.

\section{Availability of data and materials}

All relevant data are reported within the manuscript or in the cited references.

Ethics approval and consent to participate

Not applicable.

\section{Consent for publication}

Not applicable.

\section{Competing interests}

The authors declare that they have no competing interests.

\section{Author details}

${ }^{1}$ Medicine \& Rheumatology Unit, IRCCS Istituto Ortopedico Rizzoli (IOR), via Pupilli 1, 40136 Bologna, Italy. ${ }^{2}$ Department of Biomedical and Neuromotor Sciences (DIBINEM), Section of Rheumatology, University of Bologna, Bologna, Italy. ${ }^{3}$ Rheumatology Unit, Department of Biotechnological and Applied Clinical Sciences, University of L'Aquila, L'Aquila, Italy.

Received: 22 June 2020 Accepted: 3 September 2020

Published online: 28 October 2020

\section{Supplementary information}

Supplementary information accompanies this paper at https://doi.org/10. 1186/s41927-020-00165-0.

Additional file 1 Table S1. Quality assessment of randomized clinical trials. Table S2. Quality assessment of observational studies. Figure S1. Funnel plot. Meta-analysis of muscle pain as presenting symptom of COVID-19. Figure S2. Funnel plot. Meta-analysis of muscle pain prevalence during the course of COVID-19. Figure S3. Funnel plot. Metaanalysis of fatigue as presenting symptom of COVID-19. Figure S4. Funnel plot. Meta-analysis of fatigue prevalence during the course of COVID19

\section{Author details}

Supplementary information accompanies this paper at https://doi.org/10. 1186/s41927-020-00165-0. 'Medicine \& Rheumatology Unit, IRCCS Istituto Ortopedico Rizzoli (IOR), via Pupilli 1, 40136 Bologna, Italy. ${ }^{2}$ Department of Biomedical and Neuromotor Sciences (DIBINEM), Section of Rheumatology, University of Bologna, Bologna, Italy. ${ }^{3}$ Rheumatology Unit, Department of Biotechnological and Applied Clinical Sciences, University of L'Aquila,

L'Aquila, Italy.

Received: 22 June 2020 Accepted: 3 September 2020

Published online: 28 October 2020

\section{References}

1. Phelan AL, Katz R, Gostin LO. The Novel Coronavirus Originating in Wuhan, China: Challenges for Global Health Governance. JAMA. 2020;10.1001/jama. 2020.1097. https://doi.org/10.1001/jama.2020.1097.

2. Peiris JS, Yuen KY, Osterhaus AD, Stöhr K. The severe acute respiratory syndrome. N Engl J Med. 2003;349(25):2431-41.

3. Zumla A, Hui DS, Perlman S. Middle East respiratory syndrome. Lancet. 2015; 386(9997):995-1007.

4. Jiang F, Deng L, Zhang L, Cai Y, Cheung CW, Xia Z. Review of the clinical characteristics of coronavirus disease 2019 (COVID-19). J Gen Intern Med. 2020;35(5):1545-9. 
5. Verdecchia P, Cavallini C, Spanevello A, Angeli F. The pivotal link between ACE2 deficiency and SARS-CoV-2 infection. Eur I Intern Med. 2020;76:14-20.

6. Li MY, Li L, Zhang Y, Wang XS. Expression of the SARS-CoV-2 cell receptor gene ACE2 in a wide variety of human tissues. Infect Dis Poverty. 2020;9(1):45.

7. Marks M, Marks JL. Viral arthritis. Clin Med (Lond). 2016;16(2):129-34.

8. Hoang MP, Park J. Vasculitis. In: Hoang MP, Selim MA, editors. Hospitalbased Dermatopathology: an illustrated diagnostic guide. Cham: Springer International Publishing; 2020. p. 245-96.

9. Moroncini G, Mori S, Tonnini C, Gabrielli A. Role of viral infections in the etiopathogenesis of systemic sclerosis. Clin Exp Rheumatol. 2013; 31(2 Suppl 76):3-7.

10. Salvarani C, Cantini F, Boiardi L, Hunder GG. Polymyalgia rheumatica and giant-cell arteritis. N Engl J Med. 2002;347(4):261-71.

11. Moher D, Liberati A, Tetzlaff J, Altman DG. Preferred reporting items for systematic reviews and meta-analyses: the PRISMA statement. PLoS Med. 2009;6(7):e1000097.

12. Schardt C, Adams MB, Owens T, Keitz S, Fontelo P. Utilization of the PICO framework to improve searching PubMed for clinical questions. BMC Med Inform Decis Mak. 2007;7:16.

13. Higgins JP, Altman DG, Gotzsche PC, Juni P, Moher D, Oxman AD, et al. The Cochrane Collaboration's tool for assessing risk of bias in randomised trials. Bmj. 2011;343:d5928.

14. Wells GASB, O'Connell D, Peterson J, Welch V, Losos M, et al. The Newcastle-Ottawa scale (NOS) for assessing the quality of nonrandomised studies in meta-analysis; 2019. [Available from: [Website]. http://www.ohri. ca/programs/clinical_epidemiology/oxford.asp.

15. Johanna-Briggs I. Critical appraisal tools; 2017. Available from: http:// joannabriggs.org/research/critical-appraisal-tools.html.

16. Lin L, Chu H. Quantifying publication bias in meta-analysis. Biometrics. 2018; 74(3):785-94.

17. Duval $\mathrm{S}$, Tweedie R. Trim and fill: a simple funnel-plot-based method of testing and adjusting for publication bias in meta-analysis. Biometrics. 2000;56(2):455-63.

18. Redd WD, Zhou JC, Hathorn KE, et al. Prevalence and Characteristics of Gastrointestinal Symptoms in Patients With Severe Acute Respiratory Syndrome Coronavirus 2 Infection in the United States: a Multicenter Cohort Study. Gastroenterology. 2020;159(2):765-767.e2. https://doi.org/10 1053/j.gastro.2020.04.045

19. Paderno A, Schreiber A, Grammatica A, et al. Smell and taste alterations in COVID-19: a cross-sectional analysis of different cohorts. Int Forum Allergy Rhinol. 2020;10(8):955-62. https://doi.org/10.1002/alr.22610.

20. Zhou Y, He Y, Yang H, Yu H, Wang T, Chen Z, et al. Development and validation a nomogram for predicting the risk of severe COVID-19: a multicenter study in Sichuan, China. PLoS One. 2020;15(5):e0233328.

21. Chen R, Liang W, Jiang M, et al. Risk Factors of Fatal Outcome in Hospitalized Subjects With Coronavirus Disease 2019 From a Nationwide Analysis in China. Chest. 2020;158(1):97-105. https://doi.org/10.1016/j.chest. 2020.04.010

22. Chen T, Dai Z, Mo P, et al. Clinical characteristics and outcomes of older patients with coronavirus disease 2019 (COVID-19) in Wuhan, China (2019): a single-centered, retrospective study. J Gerontol A Biol Sci Med Sci. 2020; glaa089. https://doi.org/10.1093/gerona/glaa089.

23. Chen L, Deng C, Chen $X$, et al. Ocular manifestations and clinical characteristics of 535 cases of COVID-19 in Wuhan, China: a cross-sectional study. Acta Ophthalmol. 2020;10.1111/aos.14472. https://doi.org/10.1111/aos.14472.

24. Mo P, Xing Y, Xiao Y, et al. Clinical characteristics of refractory COVID-19 pneumonia in Wuhan, China. Clin Infect Dis. 2020;ciaa270. https://doi.org/10. 1093/cid/ciaa270.

25. Chen T, Wu D, Chen H, Yan W, Yang D, Chen G, et al. Clinical characteristics of 113 deceased patients with coronavirus disease 2019: retrospective study. Bmj. 2020;368:m1091.

26. Chen Y, Yang D, Cheng B, et al. Clinical Characteristics and Outcomes of Patients With Diabetes and COVID-19 in Association With Glucose-Lowering Medication. Diabetes Care. 2020;43(7):1399-407. https://doi.org/10.2337/ dc20-0660.

27. Du RH, Liu LM, Yin W, et al. Hospitalization and Critical Care of 109 Decedents with COVID-19 Pneumonia in Wuhan, China. Ann Am Thorac Soc. 2020;17(7):839-46. https://doi.org/10.1513/AnnalsATS.202003-225OC.

28. Huang R, Zhu L, Xue L, Liu L, Yan X, Wang J, et al. Clinical findings of patients with coronavirus disease 2019 in Jiangsu province, China: a retrospective, multi-center study. PLoS Negl Trop Dis. 2020;14(5):e0008280.
29. Liu F, Li L, Xu M, Wu J, Luo D, Zhu Y, et al. Prognostic value of interleukin-6, C-reactive protein, and procalcitonin in patients with COVID-19. J Clin Virol. 2020;127:104370.

30. Palaiodimos L, Kokkinidis DG, Li W, et al. Severe obesity, increasing age and male sex are independently associated with worse in-hospital outcomes, and higher in-hospital mortality, in a cohort of patients with COVID-19 in the Bronx, New York. Metabolism. 2020;108:154262.

31. Cao J, Tu WJ, Cheng W, et al. Clinical Features and Short-term Outcomes of 102 Patients with Coronavirus Disease 2019 in Wuhan, China. Clin Infect Dis. 2020;71(15):748-55. https://doi.org/10.1093/cid/ciaa243.

32. Chen $\mathrm{Q}$, Zheng Z, Zhang $\mathrm{C}$, et al. Clinical characteristics of 145 patients with corona virus disease 2019 (COVID-19) in Taizhou, Zhejiang, China. Infection. 2020;48(4):543-51. https://doi.org/10.1007/s15010-020-01432-5.

33. Hung IF, Lung KC, Tso EY, et al. Triple combination of interferon beta-1b, lopinavir-ritonavir, and ribavirin in the treatment of patients admitted to hospital with COVID-19: an open-label, randomised, phase 2 trial. Lancet. 2020;395(10238):1695-704. https:/doi.org/10.1016/S0140-6736(20)31042-4

34. Javanian M, Bayani M, Shokri M, et al. Clinical and laboratory findings from patients with COVID-19 pneumonia in Babol North of Iran: a retrospective cohort study. Rom J Intern Med. 2020;58(3):161-7. https://doi.org/10.2478/ rjim-2020-0013.

35. Ji M, Yuan L, Shen W, Lv J, Li Y, Li M, et al. Characteristics of disease progress in patients with coronavirus disease 2019 in Wuhan, China. Epidemiol Infect. 2020;148:e94.

36. Lian J, Jin X, Hao S, et al. Analysis of Epidemiological and Clinical Features in Older Patients With Coronavirus Disease 2019 (COVID-19) Outside Wuhan. Clin Infect Dis. 2020;71(15):740-7. https://doi.org/10.1093/cid/ciaa242.

37. Liguori C, Pierantozzi M, Spanetta M, et al. Subjective neurological symptoms frequently occur in patients with SARS-CoV2 infection. Brain Behav Immun. 2020;88:11-16. https://doi.org/10.1016/j.bbi.2020.05.037.

38. Liu Y, Du X, Chen J, et al. Neutrophil-to-lymphocyte ratio as an independent risk factor for mortality in hospitalized patients with COVID-19. J Infect. 2020 81(1):e6-12. https://doi.org/10.1016/j.jinf.2020.04.002.

39. Meng Y, Wu P, Lu W, Liu K, Ma K, Huang L, et al. Sex-specific clinical characteristics and prognosis of coronavirus disease-19 infection in Wuhan, China: a retrospective study of 168 severe patients. PLoS Pathog. 2020;16(4):e1008520.

40. Pan L, Mu M, Yang P, Sun Y, Wang R, Yan J, et al. Clinical characteristics of COVID-19 patients with digestive symptoms in Hubei, China: a descriptive, cross-sectional, multicenter study. Am J Gastroenterol. 2020;115(5):766-73.

41. Ren D, Ren C, Yao RQ, Feng YW, Yao YM. Clinical features and development of sepsis in patients infected with SARS-CoV-2: a retrospective analysis of 150 cases outside Wuhan, China. Intensive Care Med. 2020;46(8):1630-3. https://doi.org/10.1007/s00134-020-06084-5.

42. Shi $Q$, Zhang $X$, Jiang F, et al. Clinical Characteristics and Risk Factors for Mortality of COVID-19 Patients With Diabetes in Wuhan, China: a TwoCenter, Retrospective Study. Diabetes Care. 2020;43(7):1382-91. https://doi. org/10.2337/dc20-0598.

43. Shi S, Qin M, Shen B, et al. Association of Cardiac Injury With Mortality in Hospitalized Patients With COVID-19 in Wuhan, China. JAMA Cardiol. 2020; 5(7):802-10. https://doi.org/10.1001/jamacardio.2020.0950.

44. Wang D, Hu B, Hu C, Zhu F, Liu X, Zhang J, et al. Clinical characteristics of 138 hospitalized patients with 2019 novel coronavirus-infected pneumonia in Wuhan, China. Jama. 2020;323(11):1061-9.

45. Wang D, Yin Y, Hu C, Liu X, Zhang X, Zhou S, et al. Clinical course and outcome of 107 patients infected with the novel coronavirus, SARS-CoV-2, discharged from two hospitals in Wuhan, China. Crit Care. 2020;24(1):188.

46. Wang G, Chen W, Jin X, Chen YP. Description of COVID-19 cases along with the measures taken on prevention and control in Zhejiang, China. J Med Virol. 2020;10.1002/jmv.25906. https://doi.org/10.1002/jmv.25906.

47. Wang R, Pan M, Zhang X, Han M, Fan X, Zhao F, et al. Epidemiological and clinical features of 125 hospitalized patients with COVID-19 in Fuyang, Anhui, China. Int J Infect Dis. 2020;95:421-8.

48. Wang Z, Du Z, Zhu F. Glycosylated hemoglobin is associated with systemic inflammation, hypercoagulability, and prognosis of COVID-19 patients. Diabetes Res Clin Pract. 2020;164:108214.

49. Yang W, Cao Q, Qin L, Wang X, Cheng Z, Pan A, et al. Clinical characteristics and imaging manifestations of the 2019 novel coronavirus disease (COVID19):a multi-center study in Wenzhou city, Zhejiang, China. J Inf Secur. 2020; 80(4):388-93.

50. Zhang G, Nie S, Zhang Z, Zhang Z. Longitudinal Change of Severe Acute Respiratory Syndrome Coronavirus 2 Antibodies in Patients with 
Coronavirus Disease 2019. J Infect Dis. 2020;222(2):183-8. https://doi.org/10. 1093/infdis/jiaa229.

51. Zhang J, Yu M, Tong S, Liu LY, Tang LV. Predictive factors for disease progression in hospitalized patients with coronavirus disease 2019 in Wuhan. China J Clin Virol. 2020;127:104392.

52. Zhang Y, Li H, Zhang J, et al. The clinical characteristics and outcomes of patients with diabetes and secondary hyperglycaemia with coronavirus disease 2019: a single-centre, retrospective, observational study in Wuhan. Diabetes Obes Metab. 2020;22(8):1443-54. https://doi.org/10.1111/dom.14086.

53. Zheng F, Tang W, Li H, Huang YX, Xie YL, Zhou ZG. Clinical characteristics of 161 cases of corona virus disease 2019 (COVID-19) in Changsha. Eur Rev Med Pharmacol Sci. 2020;24(6):3404-10.

54. Zhou F, Yu T, Du R, Fan G, Liu Y, Liu Z, et al. Clinical course and risk factors for mortality of adult inpatients with COVID-19 in Wuhan, China: a retrospective cohort study. Lancet. 2020;395(10229):1054-62.

55. Zhou S, Zhu T, Wang Y, Xia L. Imaging features and evolution on CT in 100 COVID-19 pneumonia patients in Wuhan, China. Eur Radiol. 2020;30(10): 5446-54. https://doi.org/10.1007/s00330-020-06879-6.

56. Zhou X, Zhu J, Xu T. Clinical characteristics of coronavirus disease 2019 (COVID-19) patients with hypertension on renin-angiotensin system inhibitors. Clin Exp Hypertens. 2020;42(7):656-60. https://doi.org/10.1080/ 10641963.2020.1764018.

57. Zhao W, Zhong Z, Xie X, Yu Q, Liu J. Relation between chest CT findings and clinical conditions of coronavirus disease (COVID-19) pneumonia: a multicenter study. AJR Am J Roentgenol. 2020;214(5):1072-7.

58. Wu C, Chen X, Cai Y, et al. Risk Factors Associated With Acute Respiratory Distress Syndrome and Death in Patients With Coronavirus Disease 2019 Pneumonia in Wuhan, China. JAMA Intern Med. 2020;180(7):1-11. https:// doi.org/10.1001/jamainternmed.2020.0994.

59. Zhang R, Ouyang H, Fu L, et al. CT features of SARS-CoV-2 pneumonia according to clinical presentation: a retrospective analysis of 120 consecutive patients from Wuhan city. Eur Radiol. 2020;30(8):4417-26. https://doi.org/10.1007/s00330-020-06854-1.

60. Yao $Q$, Wang $P$, Wang $X$, et al. A retrospective study of risk factors for severe acute respiratory syndrome coronavirus 2 infections in hospitalized adult patients. Pol Arch Intern Med. 2020;130(5):390-9. https://doi.org/10.20452/ pamw.15312.

61. Chen J, Qi T, Liu L, Ling Y, Qian Z, Li T, et al. Clinical progression of patients with COVID-19 in Shanghai, China. J Inf Secur. 2020;80(5):e1-6.

62. Hu K, Guan WJ, Bi Y, et al. Efficacy and safety of Lianhuagingwen capsules, a repurposed Chinese herb, in patients with coronavirus disease 2019: a multicenter, prospective, randomized controlled trial. Phytomedicine. 2020; 153242.

63. Huang J, Cheng A, Lin S, Zhu Y, Chen G. Individualized prediction nomograms for disease progression in mild COVID-19. J Med Virol. 2020;10. 1002/jmv.25969. https://doi.org/10.1002/jmv.25969.

64. Hur K, Price CPE, Gray EL, et al. Factors Associated With Intubation and Prolonged Intubation in Hospitalized Patients With COVID-19. Otolaryngol Head Neck Surg. 2020;163(1):170-8. https://doi.org/10.1177/ 0194599820929640.

65. Klopfenstein T, Kadiane-Oussou NJ, Royer PY, Toko L, Gendrin V, Zayet S. Diarrhea: An underestimated symptom in Coronavirus disease 2019. Clin Res Hepatol Gastroenterol. 2020;44(3):282-3. https://doi.org/10.1016/.j.linre.2020.04.002

66. Li W, Zhang B, Lu J, et al. The characteristics of household transmission of COVID-19. Clin Infect Dis. 2020;ciaa450. https://doi.org/10.1093/cid/ciaa450

67. Nowak B, Szymański P, Pańkowski I, et al. Clinical characteristics and shortterm outcomes of patients with coronavirus disease 2019: a retrospective single-center experience of a designated hospital in Poland. Pol Arch Intern Med. 2020;130(5):407-11. https://doi.org/10.20452/pamw.15361.

68. Qi L, Yang Y, Jiang D, et al. Factors associated with the duration of viral shedding in adults with COVID-19 outside of Wuhan, China: a retrospective cohort study. Int J Infect Dis. 2020;96:531-7. https://doi.org/10.1016/j.ijid. 2020.05.045

69. Tang W, Cao Z, Han M, Wang Z, Chen J, Sun W, et al. Hydroxychloroquine in patients with mainly mild to moderate coronavirus disease 2019: open label, randomised controlled trial. Bmj. 2020;369:m1849.

70. Tian S, Hu N, Lou J, Chen K, Kang X, Xiang Z, et al. Characteristics of COVID19 infection in Beijing. J Inf Secur. 2020;80(4):401-6.

71. Yan Y, Yang Y, Wang F, et al. Clinical characteristics and outcomes of patients with severe covid-19 with diabetes. BMJ Open Diabetes Res Care. 2020;8(1):e001343. https://doi.org/10.1136/bmjdrc-2020-001343.
72. Zhang G, Hu C, Luo L, Fang F, Chen Y, Li J, et al. Clinical features and shortterm outcomes of 221 patients with COVID-19 in Wuhan, China. J Clin Virol. 2020;127:104364.

73. Zheng Y, Zhang Y, Chi H, et al. The hemocyte counts as a potential biomarker for predicting disease progression in COVID-19: a retrospective study. Clin Chem Lab Med. 2020;58(7):1106-15. https://doi.org/10.1515/ cclm-2020-0377.

74. Garazzino S, Montagnani C, Donà D, et al. Multicentre Italian study of SARSCoV-2 infection in children and adolescents, preliminary data as at 10 April 2020. Euro Surveill. 2020;25(18):2000600. https://doi.org/10.2807/1560-7917. ES.2020.25.18.2000600

75. Lu Y, Li Y, Deng W, et al. Symptomatic Infection is Associated with Prolonged Duration of Viral Shedding in Mild Coronavirus Disease 2019: a Retrospective Study of 110 Children in Wuhan. Pediatr Infect Dis J. 2020; 39(7):e95-9. https://doi.org/10.1097/INF.0000000000002729.

76. Menter T, Haslbauer JD, Nienhold R, et al. Postmortem examination of COVID-19 patients reveals diffuse alveolar damage with severe capillary congestion and variegated findings in lungs and other organs suggesting vascular dysfunction. Histopathology. 2020;10.1111/his.14134. https://doi. org/10.1111/his.14134.

77. Verdoni L, Mazza A, Gervasoni A, et al. An outbreak of severe Kawasaki-like disease at the Italian epicentre of the SARS-CoV-2 epidemic: an observational cohort study. Lancet. 2020;395(10239):1771-8. https://doi.org/ 10.1016/S0140-6736(20)31103-X.

78. Rivera-Figueroa El, Santos R, Simpson S, Garg P. Incomplete Kawasaki Disease in a Child with Covid-19. Indian Pediatr. 2020;57(7):680-1. https:// doi.org/10.1007/s13312-020-1900-0.

79. Jones VG, Mills M, Suarez D, et al. COVID-19 and Kawasaki Disease: Novel Virus and Novel Case. Hosp Pediatr. 2020;10(6):537-40. https://doi.org/10. 1542/hpeds.2020-0123.

80. Vanegas Ramirez A, Efe D, Fischer M. Drug-induced vasculitis in a patient with COVID-19. J Eur Acad Dermatol Venereol. 2020;34(8):e361-2. https:// doi.org/10.1111/jdv.16588.

81. Castelnovo L, Capelli F, Tamburello A, Faggioli PM, Mazzone A. Symmetric cutaneous vasculitis in COVID-19 pneumonia. J Eur Acad Dermatol Venereol. 2020:34(8):e362-3. https://doi.org/10.1111/jdv.16589.

82. Moeinzadeh F, Dezfouli M, Naimi A, Shahidi S, Moradi H. Newly diagnosed glomerulonephritis during COVID-19 infection undergoing immunosuppression therapy, a case report. Iran J Kidney Dis. 2020;14(3): 239-42.

83. Galván Casas C, Català A, Carretero Hernández G, et al. Classification of the cutaneous manifestations of COVID-19: a rapid prospective nationwide consensus study in Spain with 375 cases. Br J Dermatol. 2020;183(1):71-7. https://doi.org/10.1111/bjd.19163.

84. de Masson A, Bouaziz JD, Sulimovic L, et al. Chilblains is a common cutaneous finding during the COVID-19 pandemic: a retrospective nationwide study from France. J Am Acad Dermatol. 2020;83(2):667-70. https://doi.org/10.1016/j.jaad.2020.04.161.

85. Kolivras A, Dehavay F, Delplace D, et al. Coronavirus (COVID-19) infection-induced chilblains: A case report with histopathologic findings. JAAD Case Rep. 2020;6(6):489-92. https://doi.org/10.1016/j.jdcr.2020.04. 011.

86. Cordoro KM, Reynolds SD, Wattier R, McCalmont TH. Clustered cases of acral perniosis: Clinical features, histopathology, and relationship to COVID-19. Pediatr Dermatol. 2020;37(3):419-23. https://doi.org/10.1111/pde.14227.

87. Colonna C, Monzani NA, Rocchi A, Gianotti R, Boggio F, Gelmetti C. Chilblain-like lesions in children following suspected COVID-19 infection. Pediatr Dermatol. 2020;37(3):437-40. https://doi.org/10.1111/pde.14210.

88. Bouaziz JD, Duong T, Jachiet M, et al. Vascular skin symptoms in COVID-19: a french observational study. J Eur Acad Dermatol Venereol. 2020;10.1111/ jdv.16544. https://doi.org/10.1111/jdv.16544.

89. Andina D, Noguera-Morel L, Bascuas-Arribas M, et al. Chilblains in children in the setting of COVID-19 pandemic. Pediatr Dermatol. 2020;37(3):406-11. https://doi.org/10.1111/pde.14215.

90. Suarez-Valle A, Fernandez-Nieto D, Diaz-Guimaraens B, Dominguez-Santas M, Carretero I, Perez-Garcia B. Acro-ischaemia in hospitalized COVID-19 patients. J Eur Acad Dermatol Venereol. 2020;10.1111/jdv.16592. https://doi. org/10.1111/jdv. 16592.

91. Alramthan A, Aldaraji W. Two cases of COVID-19 presenting with a clinical picture resembling chilblains: first report from the Middle East. Clin Exp Dermatol. 2020;45(6):746-8. https://doi.org/10.1111/ced.14243. 
92. Zhou Y, Han T, Chen J, et al. Clinical and Autoimmune Characteristics of Severe and Critical Cases of COVID-19. Clin Transl Sci. 2020;10.1111/cts. 12805. https://doi.org/10.1111/cts.12805.

93. Zhang Y, Xiao M, Zhang S, Xia P, Cao W, Jiang W, et al. Coagulopathy and Antiphospholipid antibodies in patients with Covid-19. N Engl J Med. 2020; 382(17):e38.

94. Galeano-Valle F, Oblitas CM, Ferreiro-Mazón MM, et al. Antiphospholipid antibodies are not elevated in patients with severe COVID-19 pneumonia and venous thromboembolism. Thromb Res. 2020;192:113-5. https://doi. org/10.1016/j.thromres.2020.05.017.

95. Harzallah I, Debliquis A, Drénou B. Lupus anticoagulant is frequent in patients with Covid-19. J Thromb Haemost. 2020;18(8):2064-5. https://doi. org/10.1111/jth.14867.

96. Bowles L, Platton S, Yartey N, et al. Lupus Anticoagulant and Abnormal Coagulation Tests in Patients with Covid-19. N Engl J Med. 2020;383(3):28890. https://doi.org/10.1056/NEJMc2013656.

97. Beyrouti $R$, Adams ME, Benjamin L, et al. Characteristics of ischaemic stroke associated with COVID-19. J Neurol Neurosurg Psychiatry. 2020;91(8):889-91. https://doi.org/10.1136/jnnp-2020-323586.

98. Lazarian G, Quinquenel A, Bellal M, et al. Autoimmune haemolytic anaemia associated with COVID-19 infection. Br J Haematol. 2020;190(1):29-31. https://doi.org/10.1111/bjh.16794.

99. Lopez C, Kim J, Pandey A, Huang T, DeLoughery TG. Simultaneous onset of COVID-19 and autoimmune haemolytic anaemia. Br J Haematol. 2020;190(1): 31-2. https://doi.org/10.1111/bjh.16786

100. Bomhof G, Mutsaers PGNJ, Leebeek FWG, et al. COVID-19-associated immune thrombocytopenia. Br J Haematol. 2020;190(2):e61-4. https://doi. org/10.1111/bjh.16850

101. Zulfiqar AA, Lorenzo-Villalba N, Hassler P, Andrès E. Immune thrombocytopenic Purpura in a patient with Covid-19. N Engl J Med. 2020; 382(18):e43.

102. Li M, Nguyen CB, Yeung Z, Sanchez K, Rosen D, Bushan S. Evans syndrome in a patient with COVID-19. Br J Haematol. 2020;190(2):e59-61. https://doi. org/10.1111/bjh.16846.

103. Dogan L, Kaya D, Sarikaya T, et al. Plasmapheresis treatment in COVID-19related autoimmune meningoencephalitis: Case series. Brain Behav Immun. 2020;87:155-8. https://doi.org/10.1016/j.bbi.2020.05.022.

104. Pilotto A, Odolini S, Masciocchi S, et al. Steroid-Responsive Encephalitis in Coronavirus Disease 2019. Ann Neurol. 2020;10.1002/ana.25783. https://doi. org/10.1002/ana.25783.

105. Sedaghat Z, Karimi N. Guillain Barre syndrome associated with COVID-19 infection: a case report. J Clin Neurosci. 2020;76:233-5. https://doi.org/10. 1016/j.jocn.2020.04.062

106. Pease CT, Haugeberg G, Montague B, Hensor EM, Bhakta BB, Thomson W, et al. Polymyalgia rheumatica can be distinguished from late onset rheumatoid arthritis at baseline: results of a 5-yr prospective study. Rheumatology (Oxford). 2009;48(2):123-7.

107. Chuang TY, Hunder GG, Ilstrup DM, Kurland LT. Polymyalgia rheumatica: a 10year epidemiologic and clinical study. Ann Intern Med. 1982;97(5):672-80.

108. Dalakas MC, Hohlfeld R. Polymyositis and dermatomyositis. Lancet. 2003; 362(9388):971-82

109. Tomimitsu H, Ohta A, Nagai M, Nishina M, Ishihara S, Kohsaka H. Epidemiologic analysis of the clinical features of Japanese patients with polymyositis and dermatomyositis. Mod Rheumatol. 2016;26(3):398-402.

110. Noda K, Yoshida K, Ukichi T, Furuya K, Hirai K, Kingetsu I, et al. Myalgia in patients with Dermatomyositis and Polymyositis is attributable to fasciitis rather than myositis: a retrospective study of 32 patients who underwent Histopathological examinations. J Rheumatol. 2017;44(4):482-7.

111. Medsger TA Jr, Rodnan GP, Moossy J, Vester JW. Skeletal muscle involvement in progressive systemic sclerosis (scleroderma). Arthritis Rheum. 1968;11(4):554-68

112. Ranque B, Authier FJ, Le-Guern V, Pagnoux C, Berezne A, Allanore Y, et al. A descriptive and prognostic study of systemic sclerosis-associated myopathies. Ann Rheum Dis. 2009;68(9):1474-7.

113. Wallace D, Hahn B. Dubois' lupus Erythematosus. 7th ed. Philadelphia: Lippincott Williams \& Wilkins; 2007.

114. Guillevin L, Durand-Gasselin B, Cevallos R, Gayraud M, Lhote F, Callard P, et al. Microscopic polyangiitis: clinical and laboratory findings in eighty-five patients. Arthritis Rheum. 1999;42(3):421-30.
115. Senarathna HM, Fonseka CL, Perera HAS, De Silva PUT, Weerarathna TP. Severe disabling myalgia as an initial presentation of Polyarteritis Nodosa. Case Rep Rheumatol. 2019;2019:4364289.

116. Bahou E, Zhou L. ANCA-associated vasculitis predominantly presenting with severe myalgias. Neurol Neuroimmunol Neuroinflamm. 2017;4(4):e365.

117. Hewlett S, Choy E, Kirwan J. Furthering our understanding of fatique in rheumatoid arthritis. J Rheumatol. 2012;39(9):1775-7.

118. Overman CL, Kool MB, Da Silva JA, Geenen R. The prevalence of severe fatique in rheumatic diseases: an international study. Clin Rheumatol. 2016; 35(2):409-15.

119. Richards HL, Herrick AL, Griffin K, Gwilliam PD, Loukes J, Fortune DG. Systemic sclerosis: patients' perceptions of their condition. Arthritis Rheum. 2003:49(5):689-96.

120. Ahn GE, Ramsey-Goldman R. Fatigue in systemic lupus erythematosus. Int J Clin Rheumtol. 2012;7(2):217-27.

121. Basu N, McClean A, Harper L, Amft EN, Dhaun N, Luqmani RA, et al. Explaining fatigue in ANCA-associated vasculitis. Rheumatology (Oxford). 2013;52(9):1680-5.

122. Grayson PC, Amudala NA, McAlear CA, Leduc RL, Shereff D, Richesson R, et al. Illness perceptions and fatigue in systemic vasculitis. Arthritis Care Res (Hoboken). 2013;65(11):1835-43.

\section{Publisher's Note}

Springer Nature remains neutral with regard to jurisdictional claims in published maps and institutional affiliations.
Ready to submit your research? Choose BMC and benefit from:

- fast, convenient online submission

- thorough peer review by experienced researchers in your field

- rapid publication on acceptance

- support for research data, including large and complex data types

- gold Open Access which fosters wider collaboration and increased citations

- maximum visibility for your research: over $100 \mathrm{M}$ website views per year

At $\mathrm{BMC}$, research is always in progress.

Learn more biomedcentral.com/submissions 